International Journal of Engineering \& Technology, $7(2.12)(2018) 46-49$
International Journal of Engineering \& Technology
Website: www.sciencepubco.com/index.php/IJET
Research Paper

\title{
Panorama laser lane system based IoT
}

\author{
Gul-Won Bang ${ }^{1} *$, Yong-Ho Kim ${ }^{2}$ \\ ${ }^{1}$ Dept. of IT Auto Mobile, Gwang-ju University, Korea \\ ${ }^{2}$ Dept. of Self-designed \& Open Majors, Gwang-ju University, Korea \\ *Corresponding authorE-mail: bgcom@gwangju.ac.kr
}

\begin{abstract}
Background/Objectives: Lane marking to recognize road condition helps driver safely drive. Device in this study is developed as a complement because existing lane marking is able to difficult to identify lane according to its environment.

Methods/Statistical analysis: Lane marking has been built by spraying tiny particles of glass on a paint for the purpose of retroreflection against headlamps in automobile at night. But paint with tiny particles of glass is eliminated by automobiles and function of retro-reflection tends to lost over time. That makes lanes invisible well on rainy days at night. Panorama laser lane system based on mobile network with remote control is designed to irradiate laser beam sequentially toward the road on rainy or snowy day at night for safety guidance.

Findings: The system provides visible lane markings on rainy days or at night because laser beam lights the lane on the road to help avoid traffic accident.

Improvements/Applications: Smart road to provide safety drive will be built by the installation of system at 20 meters in the road particularly dangerous section of road or accident black spot.
\end{abstract}

Keywords: Traffic Lane; Laser Beam; IoT; Road; Safe Driving.

\section{Introduction}

Lanes on the road can be considered an essential facility to drive safely. The importance of the centerline considered as the lifeline is emphasized. But the life lane is supposed to be repainted after the first year due to faded lane. Coating material that is sprayed grains of glass on the lane to gain reflex effect by headlamps in automobiles is separated from the lane after the painting work in less than a month and that makes it impossible to acquire the reflex effect. This is a current picture of it. Laser beam irradiation on lane can contribute greatly to safety driving not only to provide outstanding visible lane marking in a rainy or snowy day at night but also to recognize curved road in the distance. Visibility of night is much better to help greatly safety driving in case of flickering laser beam on the lane.

\section{Materials and methods}

\subsection{Related works}

\subsubsection{Theory of emission and absorption in light}

Albert Einstein had found out the important principle of laser with the help of new concept introduction based on Bohr's hypothesisin interacting between atom and light, that is to say the stimulated absorption of radiation and the stimulated emission for spontaneous emission.

Spontaneous Emission: Atom in high level energy state is supposed to emit its own light which is relevant the difference of light if atom moves to low level energy state. Possibility of emitting light is entirely occurred at random.
Stimulated Emission: Light stimulated by extraneous light is emitted while atom stays high level energy state(excited state). Light that is capable of stimulating must have same wavelength of light that is capable of emitting. Coherent states between emitted light and stimulated light is called an induced emission. This process makes it possible to amplify light.

Stimulated Absorption: Atom in low level energy state is supposed to transfer high energy level condition after absorbing light. Energy difference of same light in this case has to let in light.

Substance in thermal parallel state: There are many atoms in low energy level. Some part of each atom which is composed of substance like gas, solid, and liquid where many atoms gathered is being stayed in high level energy state but almost all of atoms still tend to stay in bottom state. ${ }^{1}$

\subsubsection{Principle of laser}

Laser is an amplified light with a big amplification ratio as an exited atom or molecule starts reacting to the external stimuli. Laser is an acronym for light amplification by stimulated emission of radiation. Normal light can be made by thin lens but it spreads abroad soon. But laser which is travelling back and forth in tens of thousands inside narrow strips of tube is strong enough to go a long way without diffusing light. There are many wavelength in normal light, that is, a light in several colors mixed.Neon sign is considered relatively pure light made by electric discharge which has some wavelength thanks to Doppler effect by the atomic motion. Laser is supposed to radiate pure light with an almost same wavelength due to emitting light of resonance state. Normal light around us seems to concentrate short wave innumerably without each connection like beating drums unruly. But laser has a powerful brightness due to a lot of wave stacked one above another each otherprecisely like beating drums with a rhythm. ${ }^{2,3,4,5,6}$ 


\subsubsection{Arduino board special feature}

Main function and performance of Arduino is decided according to main chip applied. Board performance adaptive forproject is selected according to operating voltage, required number of pins, board size, price, upload method, communication support, and wireless communication with and without function. ${ }^{7}$ Arduino UNO which is popular model for first-timer is widely used. Arduino Leonards has a same performance like UNO but the difference is no converter with USB to SERIAL. In order to upload programing information in sketch, Arduino UNO is to follow converter with USB to SERIAL for inputting data into Atmega 328. Therefore, most of Arduino board is used in converter with USB to SERIAL. But Leonards is used Atmega32u4 instead of using Atmega328. Atmega32u4 built in USB controller do not need extra converter with USB to SERIAL. [Figure 1, Figure 2].

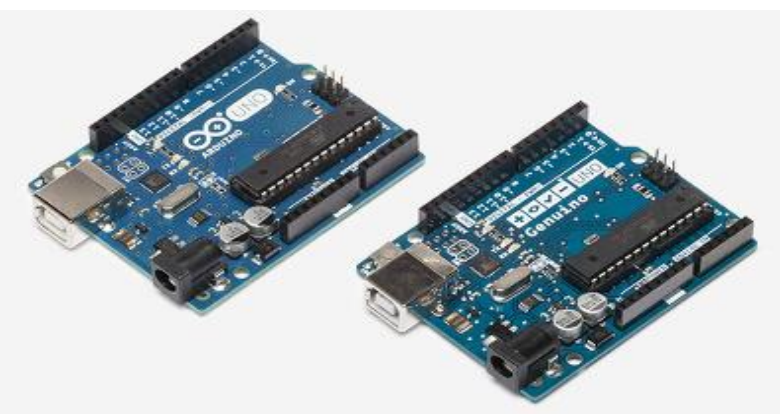

Fig. 1: Arduino UNO

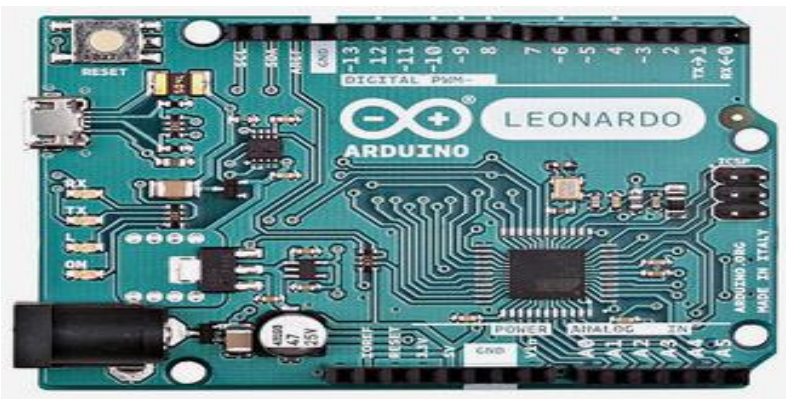

Fig. 2: Arduino LEONARDO.

Arduino Micro is used Atmega32u4 same as Leonards but big difference is small in size. It is difficult for Arduino UNO and Leonards with the size of a man's palm to make smaller size. [Figure 3].

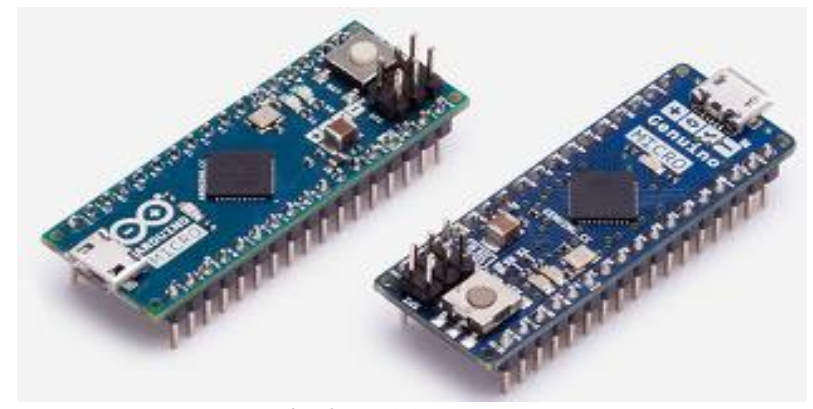

Fig. 3: Arduino MICRO.

One of characteristic in Micro and Leonards can recognize by using keyboard or mouse in Mac computers. Keyboard or mouse can be used on the condition that program allows to perform command in mouse or keyboard. That makes it possible to make a mechanical keyboard using Leonards or Micro. There arealso a small size model ArduinoNano and more compact model Arduino Mini rather than Arduino Nano. Arduino MEGA2560 which is used Atmega2560 has become the mostin Arduino series. But computing performance is same as Arduino UNO. The perfor- mance of Atmega2560 and Atmega328 is same due to operating in $16 \mathrm{MHz}$ both and difference is additional functions. [Figure 4].

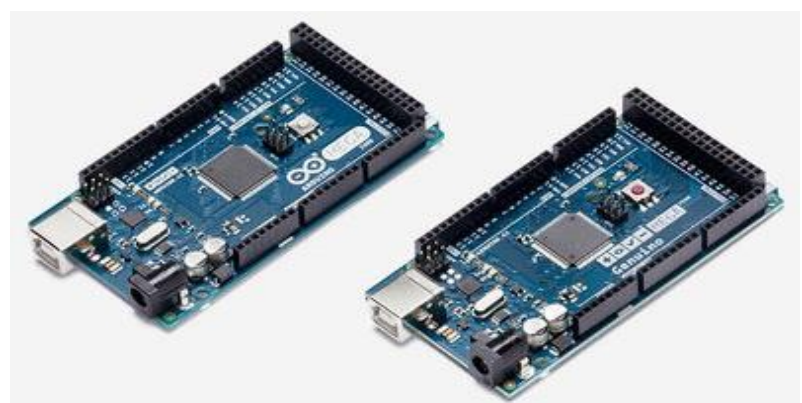

Fig. 4: Arduino MEGA 2560.

Arduino UNO can use one UART hardware, six ADC, and six PWM while Arduino Mega can use four UART hardware, sixteen ADC, and twelve PWM. It means expandability is good more than twice compared to Arduino UNO. That causes board size bigger according to good expandability. The difference between Arduino MEGA ADK and Arduino MEGA is an added model with USB Host Controller which not only can control Arduino connected Android app but can use devices which is required USB Host. Arduino ZERO which is adapted 32bit ARM Cortex M0+ core is a performance advancement model in Arduino series. Its performance is significantly improved compared to existing 8bit MCU and it is supported embedded debugger of Atmel. SAMD21 chip as a main processor has the merit of supplying USB host and device mode all together.

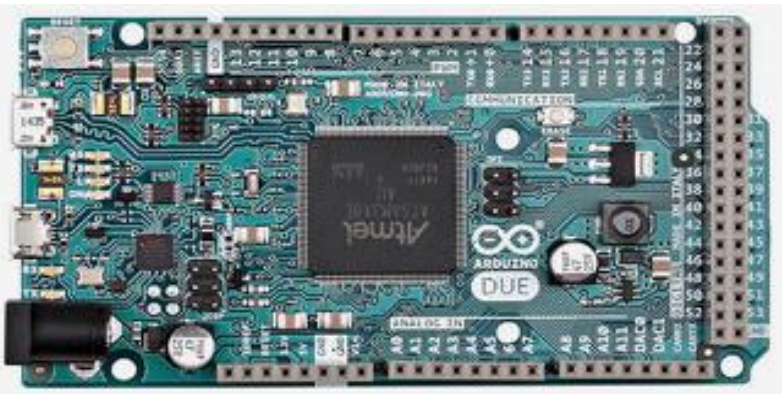

Fig. 5: Arduino DUE.

Therefore, Micro USB terminal just to left of DC Jack terminal uses USB host and device mode and can connect other device as a OTG cable utilization. The performance of Arduino DUE is very higher than UNO and Mega. Operating clock frequency is $84 \mathrm{MHz}$ and is able to analogue output due to built-in DAC with four interrupt pins and two communication pin I2C. Operating voltage is only $3.3 \mathrm{~V}$ according to GPIO output voltage with $3.3 \mathrm{~V}$. If the chip with operating voltage at $5 \mathrm{~V}$ is used or $5 \mathrm{~V}$ is applied to pin in case of using digital input or analogue input, it will be damaged. [Figure 5]

Arduino YUN unlike other Arduino is able to communicate each other through Arduino Linux and Arduino which can use simultaneously both environment in Linux and Arduino. It is also able to use internet connection due to built-in Ethernet port or WiFi module. [8].

\subsection{Proposed method}

Laser beam radiation on the road which is lane display device with a function of leading safe driving without lane departure makes it possible to provide driver with clear recognize lane owing to bad weather like rain or fog. Laser beam unit which is composed of dot type multiple laser unit is able to control angle respectively. System is composed of multiple laser beams which has a strong power due to one direction of wavelength, phase and progress direction to generate laser ray so sharply at a long distance. Laser beam radiation on the roadby multiple laser beam generator will 
be able to identify road surface and laneeasily and securely even though bad weather such as fog and heavy rainfall as well as snowfall is occurred. It is desirable that the color of laser ray is green on the side of not only protection of drivers' eyesight but clear identification of lane. Multiple color of laser ray can be provided on the condition that safe and clear identification of lane is to be secured. [9].

Controller is possible not only to turn on or off laser beam with remotely-controlled function but to turn on or off panorama type continuously. Two Arduino board are used in controller and first board is used as web server because Ethernet shield is installed for easy-to-use internet access while second board received signal from first board can turn on or off laser beam at the same time and performs the formation of panorama type laser beam which turns on multiple laser beams in serial order. This system which is used linear property of laser beam emission to road is installed guard rail for the purpose of displaying center lane to provide guide function to vehicle with safe driving for difficult road condition unable to identify center lane. [10]

Panorama laser lane system has an operation that first laser beam is turn on then next laser beam is turn on and last laser be will be turn. Next operation is that all of laser beam are supposed to turn off.This type is considered as panorama on and off type to guide safe driving in high-accident area. There are two types of operating modes. First one is automatically turn on and off mode using attached sensor responding to weather while second one is using internet which is controlled by smartphone or pc through LTE modem.

\section{Results and discussion}

Interface is designed to connect fifty laser beams. Current consumption of $5 \mathrm{~V} 200 \mathrm{~mW}$ laser beam is $210 \mathrm{~mA}$ and its power supply to turn on fifty laser beams which is used $75 \mathrm{~W}$ switching power supply with $15 \mathrm{~A}$ is $5 \mathrm{~V}$ with $10.5 \mathrm{~A}$. Microprocessor has a big memory capacity for building its own web serverand used Arduino MEGA 2560 with above fifty digital ports. It is also possible not only to connect internet through LTE modem for remote network but to control it via a smartphone or pc. [Figure 6]

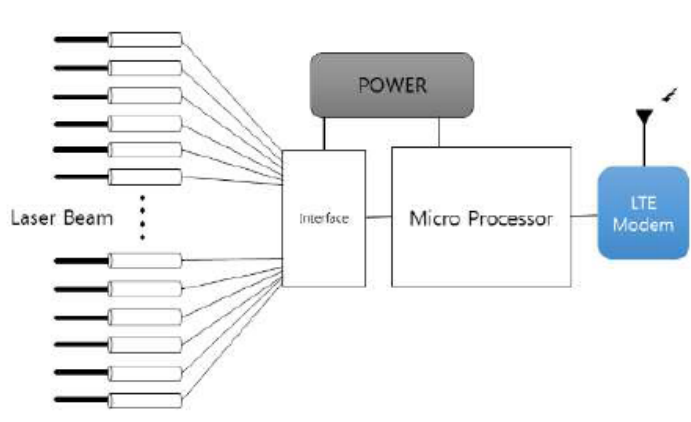

Fig. 6: Structure of Panorama Laser Lane.

Fifty laser beamsin guard rail on road verge is installed in every $20 \mathrm{~m}$ within $1 \mathrm{Km}$ and is connected control box to operate. Control picture through internet is shown in Figure7. Information of installed location is designed to operate in a variety of time for 1hour, 3hour, 6hour, and continuous hour. . [Figure 7].

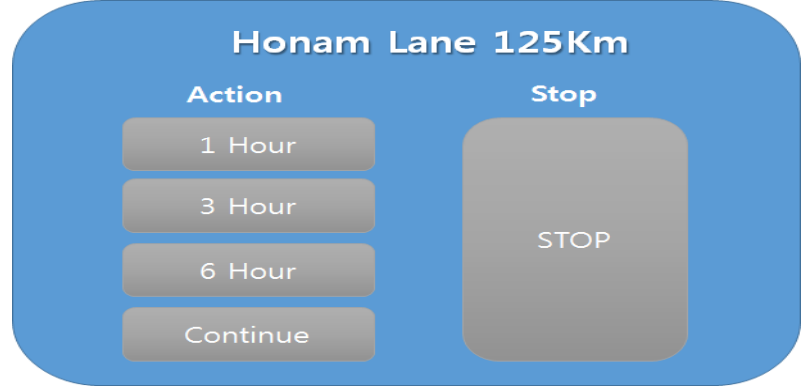

Fig. 7: Screen of Internet Control.

The comparison of panorama laser lane after/before installation is shown in figure8. [Figure 8].
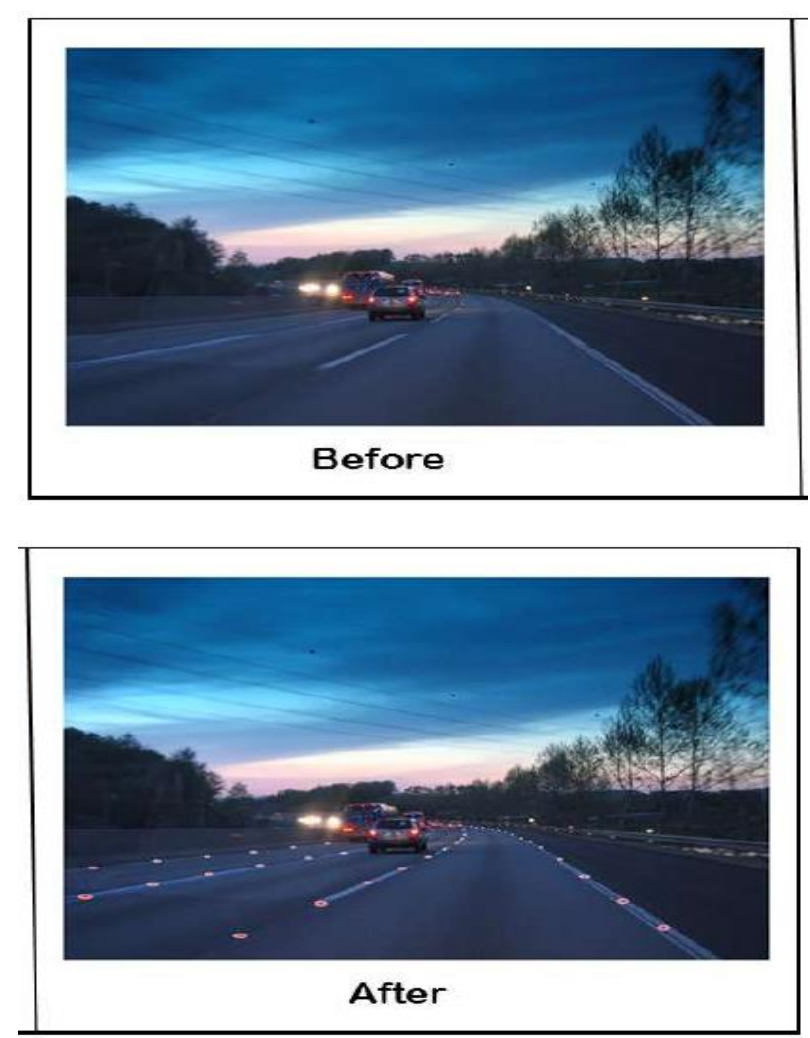

Fig. 8: Comparison of Panorama Laser Lane before and after Installation.

Clear identification of lane display installed expressway plays important roles in safety driving during rainy day or the night time. It prevents invisible lane markings especially in a rainy, snowy, and foggy day or at night to help safety driving where there is dangerous curve road or black spot in local road.

\section{Conclusion}

Existing LED range light installed guardrail in road verge has a function to display the edge of guardrail and is difficult to identify the lanes in foggy days or rainy days due to damage by vehicle and gap between display the edge of a lane and display device. Panorama laser lane system makes it possible to identify clearly not only road but lane even during bad weather conditions due to laser beam technique for safety driving. It has also safety accident prevention effect. There is an advantage not only for remote control through mobile phone and pc but no damage by vehicle but cost savings effect in installation charge and low maintenance costs due to a relatively simple components.

It is possible to install whole road with low cost. Maintenance cost is also lower. It is expected to improve economical and social effect due to induce decrease of traffic accident rate. Adding motion detection sensor helps secure driver's safety because of displaying location of pedestrians or animals who across the road in 
the night or foggy conditions. Application of multipurpose of road safety device will be able to applied, in the sense that the prediction of accident is possible in case of damaged module through not only installation in road, retaining wall, and guard rail at regular intervals but connection in additional sensors such as camera and infrared sensor as well as module.

\section{Acknowledgment}

This Study was conducted by Research funds from Gwangju University in 2018 .

\section{References}

[1] http://physica.gsnu.ac.kr/physedu/laserholo/ laserpri/laserprin.html "Laser principle", 2015.

[2] http://physica.gsnu.ac.kr/physedu/laserholo/ lasergen/lasergen.html, "Laser", 2000.

[3] http://www.fibermart.co.kr/download/board.asp? board=data\&uid=17,'Optical communication terminology", pp 6673, 2012.

[4] http://ctl.sangji.ac.kr/common/downLoad.action? siteId=biomedical\&fileSeq=228949, "Excimer Laser", 2008.

[5] Moomkyung Kim, "Second Lens design and distribution characteristics analysis for road lighting LED street lights", Bukyung University, pp.21-23. 2015.

[6] Daurelio, G, "Laser Beam Drilling Efficiency unit: the Daud", The International Society for Optical Engineering, Vil.7751 No. 2011.

[7] http://blog.naver.com/roboholic84/220585222262"To Select Arduino", 2016.

[8] http://09labs.tistory.com/5 "Arduino". 2017.

[9] Yong-Ho Kim, Gul-Won Bang, Bong-Seok Kim, Hyeong-Gyun Kim, "City-Logging System Design for Conversion City Information Service" Indian Journal of Science and Technology, 2016, 9(24), pp. 01-06

[10] Gul-Won Bang, Young-Ho Kim, "Automated Control System for Circulating Bathtub of Floating Salt Water" Indian Journal of Science and Technology, 2016, 9(24), pp. 02-03 